\title{
Qualitative behaviors of the high-order Lorenz-Stenflo chaotic system arising in mathematical physics describing the atmospheric acoustic-gravity waves
}

\author{
Guangyun Zhang ${ }^{1}$, Fuchen Zhang ${ }^{1,2^{*}}$ and Min Xiao ${ }^{3}$
}

\author{
${ }^{*}$ Correspondence: \\ zhangfuchen1983@163.com \\ ${ }^{1}$ College of Mathematics and \\ Statistics, Chongqing Technology \\ and Business University, Chongqing, \\ 400067, People's Republic of China \\ ${ }^{2}$ Mathematical post-doctoral \\ station, College of Mathematics and \\ Statistics, Southwest University, \\ Chongqing, 400716, People's \\ Republic of China \\ Full list of author information is \\ available at the end of the article
}

\begin{abstract}
The boundedness of chaotic systems plays an important role in investigating the stability of the equilibrium, estimating the Lyapunov dimension of attractors, the Hausdorff dimension of attractors, the existence of periodic solutions, chaos control, and chaos synchronization. However, as far as the authors know, there are only a few papers dealing with bounds of high-order chaotic systems due to their complex algebraic structure. To sort this out, in this paper, we study the bounds of a high-order Lorenz-Stenflo system arising in mathematical physics. Based on Lyapunov stability theory, we show that there exists a globally exponential attractive set for this system. The innovation of the paper is that we not only prove that this system is globally bounded for all the parameters, but also give a family of mathematical expressions of global exponential attractive sets of this system with respect to its parameters. We also study some other dynamical characteristics of this chaotic system such as invariant sets and chaotic behaviors. To justify the theoretical analysis, we carry out detailed numerical simulations.
\end{abstract}

Keywords: High-order Lorenz-Stenflo system; Lyapunov exponents; Lyapunov stability; domain of attraction; nonlinear dynamics

\section{Springer}

\section{Introduction}

Chaos phenomena and chaotic systems have been extensively studied by many researchers due to their various applications in the fields of atmospheric dynamics, population dynamics, electric circuits, cryptology, fluid dynamics, lasers, engineering, stock exchanges, chemical reactions, and so on [1-11]. Most of the complex dynamical phenomena are characterized by chaotic and hyperchaotic systems of nonlinear ordinary differential equations [1-21].

Stenflo [22] obtained the Lorenz-Stenflo equation from the equations describing the atmospheric acoustic-gravity waves. The Lorenz-Stenflo equation is described by the fol-

(c) The Author(s) 2017. This article is distributed under the terms of the Creative Commons Attribution 4.0 International License (http://creativecommons.org/licenses/by/4.0/), which permits unrestricted use, distribution, and reproduction in any medium, provided you give appropriate credit to the original author(s) and the source, provide a link to the Creative Commons license, and indicate if changes were made. 
lowing equations:

$$
\left\{\begin{array}{l}
\frac{d x}{d t}=\sigma(y-x)+s v, \\
\frac{d y}{d t}=-x z+r x-y, \\
\frac{d z}{d t}=x y-b z, \\
\frac{d v}{d t}=-x-\sigma v,
\end{array}\right.
$$

where $x, y, z, v$ are state variables of the Lorenz-Stenflo equation (1), and $\sigma, s, r, b$ are positive parameters of the system, $\sigma$ is the Prandtl number, $r$ is the generalized Rayleigh parameter, $b$ is the geometric parameter, and $s$ is the rotation parameter. The LorenzStenflo equation is regarded as an extended Lorenz equation since it reduces to the Lorenz equation [1] when the rotation parameter $s$ is zero, and it also can be obtained from the rotating thermal convection equations. The Lorenz-Stenflo system is a four-dimensional continuous-time dynamical system, derived to model atmospheric acoustic-gravity waves in a rotating atmosphere. Knowledge about acoustic gravity waves is important because they may be responsible both for minor local weather changes and for large-scale phenomena, for instance, storms. Many dynamical behaviors such as stability [23], bifurcation [24, 25], periodic solutions [26] and chaotic behaviors [27] of the Lorenz-Stenflo equations have been thoroughly studied for decades after Stenflo.

To improve the stability or predictability of the Lorenz-Stenflo system (1), Park et al. [26, 28] introduced the high-order Lorenz-Stenflo equations by including terms with higher vertical wave numbers:

$$
\left\{\begin{array}{l}
\frac{d x}{d t}=\sigma(y-x)+s v, \\
\frac{d y}{d t}=-x z+r x-y, \\
\frac{d z}{d t}=x y-x u-b z, \\
\frac{d v}{d t}=-x-\sigma v, \\
\frac{d u}{d t}=x z-2 x \omega-(1+2 b) u, \\
\frac{d \omega}{d t}=2 x u-4 b \omega,
\end{array}\right.
$$

where $x, y, z, v, u$, and $\omega$ are state variables, $\sigma, s, r$, and $b$ are the same positive parameters as in the original Lorenz-Stenflo system (1). Various dynamical behaviors, such as stability, periodic and chaotic solutions, and Lyapunov exponents spectra of the high-order LorenzStenflo equations (2), have been thoroughly studied [28, 29]. When $\sigma=10, b=\frac{8}{3}, r=40$, $s=50$, system (2) has a chaotic attractor [28, 29]. When $\sigma=10, b=\frac{8}{3}, r=40, s=50$, chaotic attractors of system (2) in $x O y z$ space are shown in Figure 1. Chaotic attractors of system (2) in $y O z$ plane are shown in Figure 2. Chaotic attractors of system (2) in $x O y$ plane are shown in Figure 3. Chaotic attractors of system (2) in $x O z$ plane are shown in Figure 4.

Remark 1 An oscillation in a dynamical system can be localized numerically if the initial conditions from its open neighborhood lead to the long-time behavior that approaches the oscillation. Such an oscillation (or a set of oscillations) is called an attractor, and its attracting set is called a basin of attraction. Thus, from a point of view of the numerical 


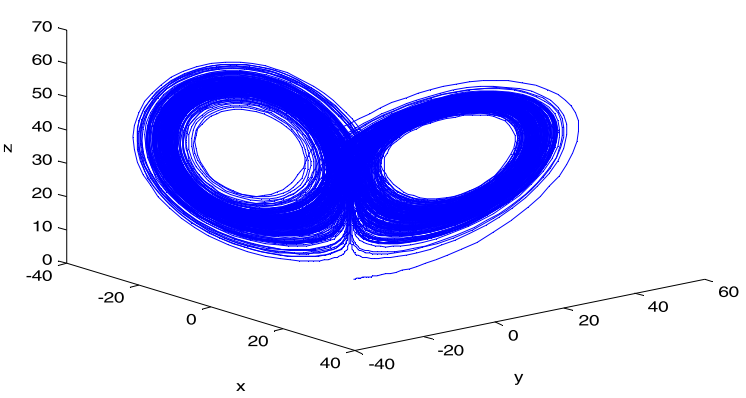

Figure 1 Chaotic attractors of system (2) in $x O y z$ space.

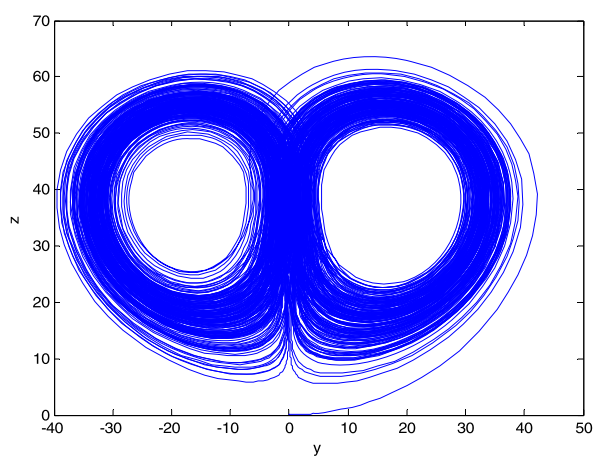

Figure 2 Chaotic attractors of system (2) in yOz plane.

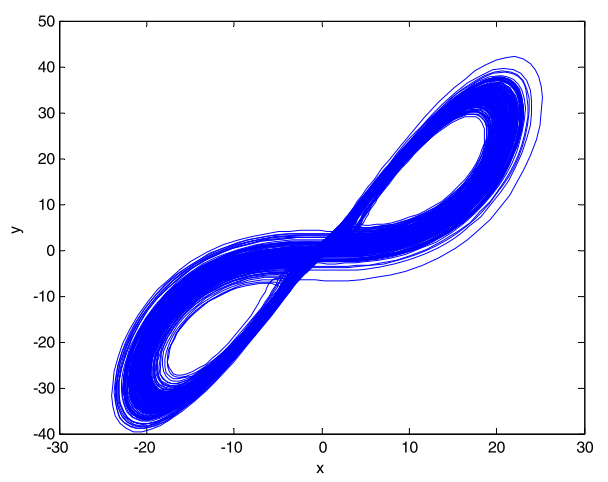

Figure 3 Chaotic attractors of system (2) in $x O y$ plane.

analysis of nonlinear dynamical models, it is essential to classify an attractor as a selfexcited or hidden attractor depending on simplicity of finding its basin of attraction [14, 30, 31]: An attractor is called a self-excited attractor if its basin of attraction intersects with an arbitrarily small open neighborhood of an unstable equilibrium; otherwise, it is called a hidden attractor (see [14] and [30,31] for a detailed discussion of the attractors in dynamical systems). Although the authors in $[28,29]$ point out that system (2) has a attractor for $\sigma=10, b=\frac{8}{3}, r=40, s=50$, they do not point out which type of attractor 
Figure 4 Chaotic attractors of system (2) in $x O z$ plane.

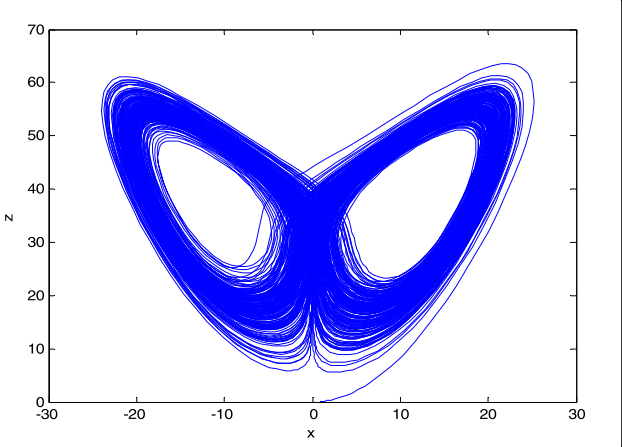

system (2) has. It is necessary and interesting to discuss the classification of the attractors in system (2) in the future.

\section{Some dynamics of high-order Lorenz-Stenflo system}

\subsection{Invariance}

The positive $z$-axis, $u$-axis, and $\omega$-axis are invariant under the flow, that is, they are positively invariant under the flow generated by system (2). However, this is not the case on the positive $x$-axis, $y$-axis, and $v$-axis for system (2) since they are all not positively invariant under the flow generated by system (2).

\subsection{Ultimate bound set and domain of attraction}

In this section, we further investigate the ultimate bound set and global domain of attraction of the high-order Lorenz-Stenflo system (2). The main result is described by the following theorems, Theorems 1 and 2 .

Theorem 1 For any $\lambda_{1}>0, m>0, \sigma>0, s>0, r>0, b>0$, there exists a positive number $M>0$ such that

$$
\Psi=\left\{X \mid \lambda_{1}\left(x-m_{2}\right)^{2}+m y^{2}+m\left(z-2 \lambda_{2}\right)^{2}+\lambda_{1} s\left(v-m_{3}\right)^{2}+m u^{2}+m\left(\omega-\lambda_{2}\right)^{2} \leq M\right\}
$$

is the ultimate bound and positively invariant set of the high-order Lorenz-Stenflo system $(2)$, where $X(t)=(x(t), y(t), z(t), v(t), u(t), \omega(t))$.

Proof Define the following Lyapunov-like function

$$
V(X)=\lambda_{1}\left(x-m_{2}\right)^{2}+m y^{2}+m\left(z-2 \lambda_{2}\right)^{2}+\lambda_{1} s\left(v-m_{3}\right)^{2}+m u^{2}+m\left(\omega-\lambda_{2}\right)^{2}
$$

where $\forall \lambda_{1}>0, \forall m>0, \lambda_{2}=\frac{\lambda_{1} \sigma+m r}{2 m}, X(t)=(x(t), y(t), z(t), v(t), u(t), \omega(t))$, and $m_{2} \in R$, $m_{3} \in R$ are arbitrary constants.

We have

$$
\begin{aligned}
& \left.\frac{d V(X(t))}{d t}\right|_{(2)} \\
& =2 \lambda_{1}\left(x-m_{2}\right) \frac{d x}{d t}+2 m y \frac{d y}{d t}+2 m\left(z-2 \lambda_{2}\right) \frac{d z}{d t}+2 \lambda_{1} s\left(v-m_{3}\right) \frac{d v}{d t} \\
& \quad+2 m u \frac{d u}{d t}+2 m\left(\omega-\lambda_{2}\right) \frac{d \omega}{d t}
\end{aligned}
$$




$$
\begin{aligned}
= & 2 \lambda_{1}\left(x-m_{2}\right)(\sigma y-\sigma x+s v)+2 m y(-x z+r x-y)+2 m\left(z-2 \lambda_{2}\right)(x y-x u-b z) \\
& +2 \lambda_{1} s\left(v-m_{3}\right)(-x-\sigma v)+2 m u[x z-2 x \omega-(1+2 b) u]+2 m\left(\omega-\lambda_{2}\right)(2 x u-4 b \omega) \\
= & -2 \lambda_{1} \sigma x^{2}+2 \lambda_{1} \sigma m_{2} x+2 \lambda_{1} s m_{3} x-2 m y^{2}-2 \lambda_{1} \sigma m_{2} y-2 b m z^{2}+4 b m \lambda_{2} z-2 \lambda_{1} s \sigma v^{2} \\
& -2 \lambda_{1} s m_{2} v+2 \lambda_{1} s m_{3} \sigma v-2 m(1+2 b) u^{2}-8 b m \omega^{2}+8 b m \lambda_{2} \omega .
\end{aligned}
$$

Let $\frac{d V(X(t))}{d t}=0$. Then, we get that the surface

$$
\Gamma:\left\{\begin{array}{l}
X \mid-2 \lambda_{1} \sigma x^{2}+2 \lambda_{1} \sigma m_{2} x+2 \lambda_{1} s m_{3} x-2 m y^{2} \\
\quad-2 \lambda_{1} \sigma m_{2} y-2 b m z^{2}+4 b m \lambda_{2} z-2 \lambda_{1} s \sigma v^{2} \\
\quad-2 \lambda_{1} s m_{2} v+2 \lambda_{1} s m_{3} \sigma v-2 m(1+2 b) u^{2}-8 b m \omega^{2}+8 b m \lambda_{2} \omega=0
\end{array}\right\}
$$

is an ellipsoid in $R^{6} \forall \lambda_{1}>0, m>0, \sigma>0, s>0, r>0, b>0$. Outside $\Gamma, \frac{d V(X(t))}{d t}<0$, whereas inside $\Gamma, \frac{d V(X(t))}{d t}>0$. Thus, the ultimate boundedness for system (2) can only be reached on $\Gamma$. Since the Lyapunov-like function $V(X)$ is a continuous function and $\Gamma$ is a bounded closed set, the function (3) can reach its maximum value $\max _{X \in \Gamma} V(X)=M$ on the surface $\Gamma$. Obviously, $\left\{X \mid V(X) \leq \max _{X \in \Gamma} V(X)=M, X \in \Gamma\right\}$ contains solutions of system (2). It is obvious that the set $\Psi$ is the ultimate bound set and positively invariant set for system (2).

This completes the proof.

Theorem 1 points that the trajectories of system (2) are ultimately bounded. However, Theorem 1 does not give the rate of the trajectories of system (2) going from the exterior of the trapping set to the interior of the trapping set. The rate of the trajectories rate of system (2) is studied in the next theorem, Theorem 2.

In the following section, we further investigate the globally attractive set of the highorder Lorenz-Stenflo system (2). We use the following Lyapunov-like function

$$
V(X)=\lambda_{1}\left(x-m_{2}\right)^{2}+m y^{2}+m\left(z-2 \lambda_{2}\right)^{2}+\lambda_{1} s\left(v-m_{3}\right)^{2}+m u^{2}+m\left(\omega-\lambda_{2}\right)^{2}
$$

which is obviously positive definite and radially unbounded. Here, $\forall \lambda_{1}>0, \forall m>0, \lambda_{2}=$ $\frac{\lambda_{1} \sigma+m r}{2 m}$, and $m_{2} \in R, m_{3} \in R$ are arbitrary constants.

Let $X(t)=(x(t), y(t), z(t), v(t), u(t), \omega(t))$ be an arbitrary solution of system (2). We have the following results for system (2).

Theorem 2 Suppose that $\forall \sigma>0, s>0, r>0, b>0$, and let

$$
\begin{aligned}
& L^{2}=\frac{1}{\theta}\left[\frac{\lambda_{1} s^{2}\left(m_{3}\right)^{2}}{\sigma}+\frac{\left(\lambda_{1} \sigma m_{2}\right)^{2}}{m}+\frac{\lambda_{1} s\left(m_{2}\right)^{2}}{\sigma}+\lambda_{1} \sigma\left(m_{2}\right)^{2}+8 b m\left(\lambda_{2}\right)^{2}+\lambda_{1} s \sigma\left(m_{3}\right)^{2}\right], \\
& \theta=\min (\sigma, b)>0 .
\end{aligned}
$$

Then, for system (2), we have the estimate

$$
\left[V(X(t))-L^{2}\right] \leq\left[V\left(X\left(t_{0}\right)\right)-L^{2}\right] e^{-\theta\left(t-t_{0}\right)} .
$$

Thus $\Omega=\left\{X \mid V(X) \leq L^{2}\right\}$ is a globally exponential attractive set of system (2), that is, $\varlimsup_{t \rightarrow+\infty} V(X(t)) \leq L^{2}$. 
Proof Define the following functions:

$$
f(x)=-\lambda_{1} \sigma x^{2}+2 \lambda_{1} s m_{3} x, \quad h(y)=-m y^{2}-2 \lambda_{1} \sigma m_{2} y, \quad g(v)=-\lambda_{1} s \sigma v^{2}-2 \lambda_{1} m_{2} s \nu .
$$

Then we have

$$
\max _{x \in R} f(x)=\frac{\lambda_{1} s^{2}\left(m_{3}\right)^{2}}{\sigma}, \quad \max _{y \in R} h(y)=\frac{\left(\lambda_{1} \sigma m_{2}\right)^{2}}{m}, \quad \max _{v \in R} g(v)=\frac{\lambda_{1} s\left(m_{2}\right)^{2}}{\sigma} .
$$

Differentiating the Lyapunov-like function $V(X)$ in (5) with respect time $t$ along the trajectory of system (2) yields

$$
\begin{aligned}
& \left.\frac{d V(X(t))}{d t}\right|_{(2)} \\
& =2 \lambda_{1}\left(x-m_{2}\right) \frac{d x}{d t}+2 m y \frac{d y}{d t}+2 m\left(z-2 \lambda_{2}\right) \frac{d z}{d t}+2 \lambda_{1} s\left(v-m_{3}\right) \frac{d v}{d t} \\
& +2 m u \frac{d u}{d t}+2 m\left(\omega-\lambda_{2}\right) \frac{d \omega}{d t} \\
& =2 \lambda_{1}\left(x-m_{2}\right)(\sigma y-\sigma x+s v)+2 m y(-x z+r x-y)+2 m\left(z-2 \lambda_{2}\right)(x y-x u-b z) \\
& +2 \lambda_{1} s\left(v-m_{3}\right)(-x-\sigma v)+2 m u[x z-2 x \omega-(1+2 b) u]+2 m\left(\omega-\lambda_{2}\right)(2 x u-4 b \omega) \\
& =-2 \lambda_{1} \sigma x^{2}+2 \lambda_{1} \sigma m_{2} x \\
& +2 \lambda_{1} s m_{3} x-2 m y^{2}-2 \lambda_{1} \sigma m_{2} y-2 b m z^{2}+4 b m \lambda_{2} z-2 \lambda_{1} s \sigma v^{2} \\
& -2 \lambda_{1} s m_{2} v+2 \lambda_{1} s m_{3} \sigma v-2 m(1+2 b) u^{2}-8 b m \omega^{2}+8 b m \lambda_{2} \omega \\
& =-\lambda_{1} \sigma x^{2}+2 \lambda_{1} \sigma m_{2} x-\lambda_{1} \sigma x^{2} \\
& +2 \lambda_{1} s m_{3} x-m y^{2}-m y^{2}-2 \lambda_{1} \sigma m_{2} y-2 b m z^{2}+4 b m \lambda_{2} z \\
& -\lambda_{1} s \sigma v^{2}+2 \lambda_{1} s m_{3} \sigma v-\lambda_{1} s \sigma v^{2}-2 \lambda_{1} s m_{2} v-2 m(1+2 b) u^{2}-8 b m \omega^{2}+8 b m \lambda_{2} \omega \\
& =-\lambda_{1} \sigma x^{2}+2 \lambda_{1} \sigma m_{2} x+f(x)-m y^{2}+h(y)-2 b m z^{2}+4 b m \lambda_{2} z-\lambda_{1} s \sigma v^{2}+2 \lambda_{1} s m_{3} \sigma v \\
& +g(v)-2 m(1+2 b) u^{2}-8 b m \omega^{2}+8 b m \lambda_{2} \omega \\
& \leq-\lambda_{1} \sigma x^{2}+2 \lambda_{1} \sigma m_{2} x+f(x)-m y^{2}+h(y)-b m z^{2}+4 b m \lambda_{2} z-\lambda_{1} s \sigma v^{2}+2 \lambda_{1} s m_{3} \sigma v \\
& +g(v)-2 m(1+2 b) u^{2}-8 b m \omega^{2}+8 b m \lambda_{2} \omega \\
& =-\lambda_{1} \sigma\left(x-m_{2}\right)^{2}+\lambda_{1} \sigma\left(m_{2}\right)^{2}+f(x)-m y^{2}+h(y)-b m\left(z-2 \lambda_{2}\right)^{2}+4 b m\left(\lambda_{2}\right)^{2} \\
& -\lambda_{1} s \sigma\left(v-m_{3}\right)^{2}+\lambda_{1} s \sigma\left(m_{3}\right)^{2}+g(v)-2 m(1+2 b) u^{2}-8 b m \omega^{2}+8 b m \lambda_{2} \omega \\
& =-\lambda_{1} \sigma\left(x-m_{2}\right)^{2}-m y^{2}-b m\left(z-2 \lambda_{2}\right)^{2} \\
& -\lambda_{1} s \sigma\left(v-m_{3}\right)^{2}-2 m(1+2 b) u^{2}-8 b m \omega^{2}+8 b m \lambda_{2} \omega \\
& +f(x)+h(y)+g(v)+\lambda_{1} \sigma\left(m_{2}\right)^{2}+4 b m\left(\lambda_{2}\right)^{2}+\lambda_{1} s \sigma\left(m_{3}\right)^{2} \\
& \leq-\lambda_{1} \sigma\left(x-m_{2}\right)^{2}-m y^{2}-b m\left(z-2 \lambda_{2}\right)^{2}-\lambda_{1} s \sigma\left(v-m_{3}\right)^{2}-m u^{2}-4 b m \omega^{2}+8 b m \lambda_{2} \omega \\
& +f(x)+h(y)+g(v)+\lambda_{1} \sigma\left(m_{2}\right)^{2}+4 b m\left(\lambda_{2}\right)^{2}+\lambda_{1} s \sigma\left(m_{3}\right)^{2} \\
& =-\lambda_{1} \sigma\left(x-m_{2}\right)^{2}-m y^{2}-b m\left(z-2 \lambda_{2}\right)^{2}-\lambda_{1} s \sigma\left(v-m_{3}\right)^{2}-m u^{2}-4 b m\left(\omega-\lambda_{2}\right)^{2}
\end{aligned}
$$




$$
\begin{aligned}
& +f(x)+h(y)+g(v)+\lambda_{1} \sigma\left(m_{2}\right)^{2}+4 b m\left(\lambda_{2}\right)^{2}+\lambda_{1} s \sigma\left(m_{3}\right)^{2}+4 b m\left(\lambda_{2}\right)^{2} \\
\leq & -\lambda_{1} \sigma\left(x-m_{2}\right)^{2}-m y^{2}-b m\left(z-2 \lambda_{2}\right)^{2}-\lambda_{1} s \sigma\left(v-m_{3}\right)^{2}-m u^{2}-b m\left(\omega-\lambda_{2}\right)^{2} \\
& +f(x)+h(y)+g(v)+\lambda_{1} \sigma\left(m_{2}\right)^{2}+8 b m\left(\lambda_{2}\right)^{2}+\lambda_{1} s \sigma\left(m_{3}\right)^{2} \\
\leq & -\theta V(X)+\max _{x \in R} f(x)+\max _{y \in R} h(y)+\max _{v \in R} g(v)+\lambda_{1} \sigma\left(m_{2}\right)^{2}+8 b m\left(\lambda_{2}\right)^{2}+\lambda_{1} s \sigma\left(m_{3}\right)^{2} \\
= & -\theta V(X)+\frac{\lambda_{1} s^{2}\left(m_{3}\right)^{2}}{\sigma}+\frac{\left(\lambda_{1} \sigma m_{2}\right)^{2}}{m}+\frac{\lambda_{1} s\left(m_{2}\right)^{2}}{\sigma} \\
& +\lambda_{1} \sigma\left(m_{2}\right)^{2}+8 b m\left(\lambda_{2}\right)^{2}+\lambda_{1} s \sigma\left(m_{3}\right)^{2} \\
= & -\theta\left[V(X(t))-L^{2}\right] .
\end{aligned}
$$

Thus, we have

$$
\left[V(X(t))-L^{2}\right] \leq\left[V\left(X\left(t_{0}\right)\right)-L^{2}\right] e^{-\theta\left(t-t_{0}\right)} .
$$

Therefore,

$$
\varlimsup_{t \rightarrow+\infty} V(X(t)) \leq L^{2}
$$

which clearly shows that $\Omega=\left\{X \mid V(X) \leq L^{2}\right\}$ is a globally exponential attractive set of system (2). The proof is complete.

Remark 2 (i) Let us take $\lambda_{1}=1, m=1, m_{2}=0, m_{3}=0$ in Theorem 2 . Then we get that

$$
\Delta=\left\{(x, y, z, v, u, w) \mid x^{2}+y^{2}+(z-\sigma-r)^{2}+s v^{2}+u^{2}+\left(\omega-\frac{\sigma+r}{2}\right)^{2} \leq \frac{2 b(\sigma+r)^{2}}{\min (\sigma, b)}\right\}
$$

is a globally exponential attractive set of system (2) according to Theorem 2 .

(ii) Taking $\sigma=10, b=\frac{8}{3}, r=40, s=50$, we get that

$$
\Delta=\left\{(x, y, z, v, u, w) \mid x^{2}+y^{2}+(z-50)^{2}+50 v^{2}+u^{2}+(\omega-25)^{2} \leq(50 \sqrt{2})^{2}\right\}
$$

is a globally exponential attractive set of system (2) according to Theorem 2. Figure 5 shows chaotic attractors of system (2) in the $(x, y, z)$ space defined by $\Delta$ in (8). Figure 6 shows

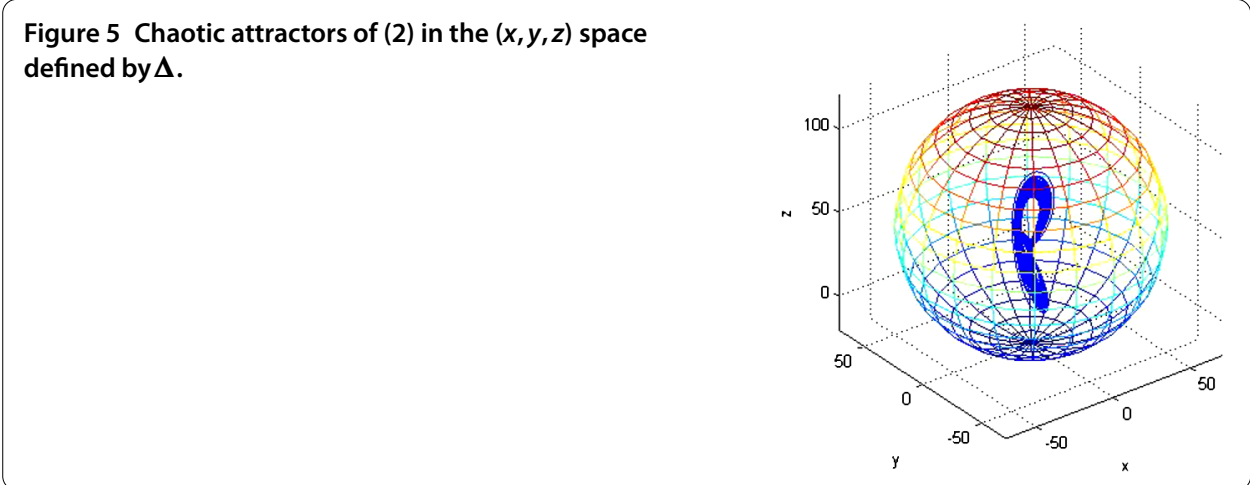




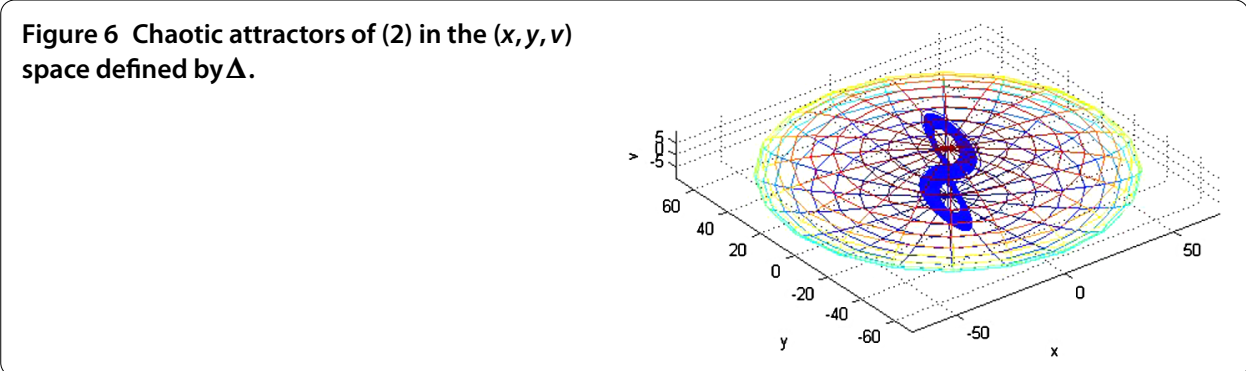

Figure 7 Chaotic attractors of (2) in the $(x, y, u)$ space defined by $\Delta$.

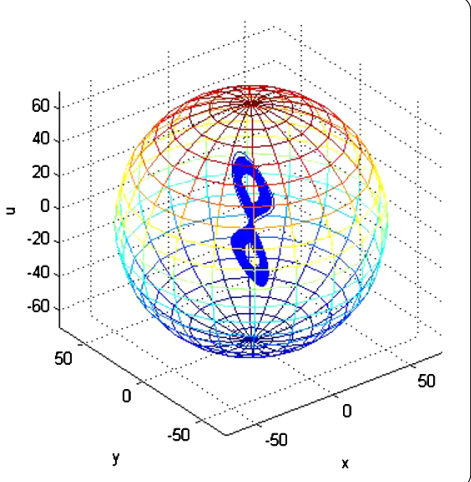

Figure 8 Chaotic attractors of $(2)$ in the $(x, y, \omega)$ space defined by $\Delta$.

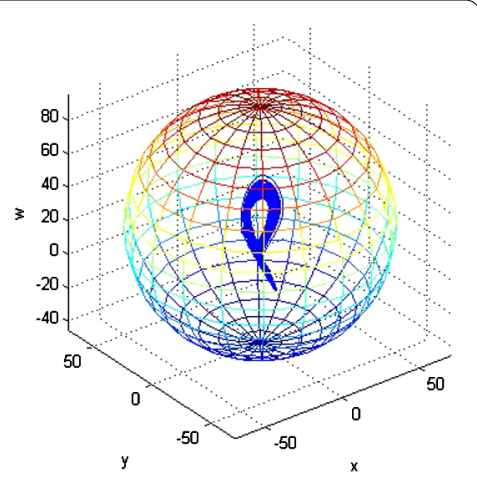

chaotic attractors of system (2) in the $(x, y, v)$ space defined by $\Delta$ in (8). Figure 7 shows chaotic attractors of system (2) in the $(x, y, u)$ space defined by $\Delta$ in (8). Figure 8 shows chaotic attractors of system (2) in the $(x, y, \omega)$ space defined by $\Delta$ in (8).

\section{Conclusions}

By means of Lyapunov-like functions, we have studied some dynamical behaviors of a high-order Lorenz-Stenflo system using theoretical analysis and numerical simulations. The obtained results show that this system has complex dynamics and this system deserves a further detailed investigation. The results of this paper are useful in many engineering applications such as chaos synchronization, chaos cryptology, coding information, and information compression. 


\section{Acknowledgements}

Fuchen Zhang is supported by National Natural Science Foundation of China (Grant No. 11501064), the Basic and Advanced Research Project of CQCSTC (Grant No. cstc2014jcyjA00040), the Scientific and Technological Research Program of Chongqing Municipal Education Commission (Grant No. KJ1500605), the Research Fund of Chongqing Technology and Business University (Grant No. 2014-56-11), China Postdoctoral Science Foundation (Grant No. 2016M590850). We thank professors Jinhu Lu in Chinese Academy of Sciences, Xiaofeng Liao in College of Electronic and Information Engineering of Southwest University, Gaoxiang Yang in Ankang University and Ping Zhou in Chongqing University of Posts and Telecommunications for their help. The authors wish to thank the editors and reviewers for their conscientious reading of this paper and numerous comments for improvement, which were extremely useful and helpful in modifying the paper.

\section{Competing interests}

The authors declare that they have no competing interests.

\section{Authors' contributions}

All authors read and approved the final manuscript.

\section{Author details}

'College of Mathematics and Statistics, Chongqing Technology and Business University, Chongqing, 400067, People's Republic of China. ${ }^{2}$ Mathematical post-doctoral station, College of Mathematics and Statistics, Southwest University, Chongqing, 400716, People's Republic of China. ${ }^{3}$ College of Automation, Nanjing University of Posts and

Telecommunications, Nanjing, 210003, People's Republic of China.

\section{Publisher's Note}

Springer Nature remains neutral with regard to jurisdictional claims in published maps and institutional affiliations.

Received: 7 June 2017 Accepted: 6 September 2017 Published online: 15 September 2017

\section{References}

1. Lorenz, EN: Deterministic non-periods flows. J. Atmos. Sci. 20, 130-141 (1963)

2. Zhang, FC, Zhang, GY: Further results on ultimate bound on the trajectories of the Lorenz system. Qual. Theory Dyn. Syst. 15(1), 221-235 (2016)

3. Li, TY, Yorke, JA: Period three implies chaos. Am. Math. Mon. 82, 985-992 (1975)

4. Bao, H, Park, JH, Cao, JD: Synchronization of fractional-order delayed neural networks with hybrid coupling. Complexity 21(S1), 106-112 (2016)

5. Matouk, AE: Chaos synchronization of a fractional-order modified Van der Pol-Duffing system via new linear control, backstepping control and Takagi-Sugeno fuzzy approaches. Complexity 21(S1), 116-124 (2016)

6. Elsayed, EM, Ahmed, AM: Dynamics of a three-dimensional systems of rational difference equations. Math. Methods Appl. Sci. 39(5), 1026-1038 (2016)

7. Kuznetsov, N, Mokaev, T, Vasilyev, P: Numerical justification of Leonov conjecture on Lyapunov dimension of Rössler attractor. Commun. Nonlinear Sci. Numer. Simul. 19(4), 1027-1034 (2014)

8. Leonov, G: Bounds for attractors and the existence of homoclinic orbits in the Lorenz system. J. Appl. Math. Mech. 65(1), 19-32 (2001)

9. Leonov, G, Bunin, A, Koksch, N: Attractor localization of the Lorenz system. Z. Angew. Math. Mech. 67, 649-656 (1987)

10. Leonov, G: General existence conditions of homoclinic trajectories in dissipative systems. Lorenz, Shimizu-Morioka, Lu and Chen systems. Phys. Lett. A 376, 3045-3050 (2012)

11. Leonov, G, Kuznetsov, K: Hidden attractors in dynamical systems. From hidden oscillations in Hilbert-Kolmogorov, Aizerman, and Kalman problems to hidden chaotic attractor in Chua circuits. Int. J. Bifurc. Chaos Appl. Sci. Eng. 23 Article ID 1330002 (2013)

12. Leonov, G, Kuznetsov, N, Kiseleva, M, Solovyeva, E, Zaretskiy, A: Hidden oscillations in mathematical model of drilling system actuated by induction motor with a wound rotor. Nonlinear Dyn. 77, 277-288 (2014)

13. Wang, XY, Wang, MJ: A hyperchaos generated from Lorenz system. Physica A 387(14), 3751-3758 (2008)

14. Leonov, G, Kuznetsov, N, Mokaev, T: Homoclinic orbits, and self-excited and hidden attractors in a Lorenz-like system describing convective fluid motion. Eur. Phys. J. Spec. Top. 224(8), 1421-1458 (2015)

15. Leonov, G, Kuznetsov, N: On differences and similarities in the analysis of Lorenz, Chen, and Lu systems. Appl. Math. Comput. 256, 334-343 (2015)

16. Zhang, FC, Liao, XF, Mu, CL: Dynamical analysis of the permanent-magnet synchronous motor chaotic system. Adv. Differ. Equ. 2017(1), Article ID 76 (2017). doi:10.1186/s13662-017-1118-1

17. Zhang, FC, Liao, XF, Zhang, GY: Qualitative behaviors of the continuous-time chaotic dynamical systems describing the interaction of waves in plasma. Nonlinear Dyn. 88(3), 1623-1629 (2017)

18. Zhang, FC, Mu, CL, Zhou, SM, Zheng, P: New results of the ultimate bound on the trajectories of the family of the Lorenz systems. Discrete Contin. Dyn. Syst., Ser. B 20(4), 1261-1276 (2015)

19. Zhang, FC, Liao, XF, Mu, CL, Zhang, GY, Chen, YA: On global boundedness of the Chen system. Discrete Contin. Dyn. Syst., Ser. B 22(4), 1673-1681 (2017)

20. Zhang, FC, Liao, XF, Zhang, GY, Mu, CL: Dynamical analysis of the generalized Lorenz systems. J. Dyn. Control Syst. 23(2), 349-362 (2017)

21. Zhang, FC, Wang, XY, Liao, XF, Zhang, GY, Mu, CL: Dynamical behaviors of a modified Lorenz-Stenflo chaotic system. Int. J. Bifurc. Chaos Appl. Sci. Eng. 27(5), Article ID 1750074 (2017)

22. Stenflo, L: Generalized Lorenz equations for acoustic-gravity waves in the atmosphere. Phys. Scr. 53(1), 83-84 (1996)

23. Yu, MY, Yang, B: Periodic and chaotic solutions of the generalized Lorenz equations. Phys. Scr. 54(2), 140-142 (1996) 
24. Yu, MY, Zhou, CT, Lai, CH: The bifurcation characteristics of the generalized Lorenz equations. Phys. Scr. 54(4), 321-324 (1996)

25. Zhou, C, Lai, CH, Yu, MY: Bifurcation behavior of the generalized Lorenz equations at large rotation numbers. J. Math. Phys. 38(10), 5225-5239 (1997). doi:10.1063/1.531938

26. Park, J, Lee, H, Jeon, YL, Baik, JJ: Periodicity of the Lorenz-Stenflo equations. Phys. Scr. 90(6), Article ID 065201 (2015)

27. Paulo, CR: On the dynamics in parameter planes of the Lorenz-Stenflo system. Phys. Scr. 90(11), Article ID 115201 (2015)

28. Park, J, Han, BS, Lee, H, Jeon, YL, Baik, JJ: Stability and periodicity of high-order Lorenz-Stenflo equations. Phys. Scr. 91(6), Article ID 065202 (2016)

29. Rech, PC: On the dynamics of a high-order Lorenz-Stenflo system. Phys. Scr. 91(12), Article ID 125201 (2016)

30. Kuznetsov, NV, Leonov, GA, Yuldashev, MV, Yuldashev, RV: Hidden attractors in dynamical models of phase-locked loop circuits: limitations of simulation in MATLAB and SPICE. Commun. Nonlinear Sci. Numer. Simul. 51, 39-49 (2017)

31. Leonov, GA, Kuznetsov, NV, Korzhemanova, NA, Kusakin, DV: Lyapunov dimension formula for the global attractor of the Lorenz system. Commun. Nonlinear Sci. Numer. Simul. 41, 84-103 (2016)

\section{Submit your manuscript to a SpringerOpen ${ }^{\circ}$ journal and benefit from:}

- Convenient online submission

- Rigorous peer review

- Open access: articles freely available online

- High visibility within the field

- Retaining the copyright to your article

Submit your next manuscript at $\gg$ springeropen.com 